\title{
Min-Switching Local Stabilization for Discrete-Time Switching Systems with Nonlinear Modes
}

\author{
Marc Jungers $^{\mathrm{a}, \mathrm{b}}$, Carlos A. C. Gonzaga ${ }^{\mathrm{a}, \mathrm{b}}$, Jamal Daafouz ${ }^{\mathrm{a}, \mathrm{b}, \mathrm{c}}$ \\ ${ }^{a}$ Université de Lorraine, CRAN, UMR 7032, Vandoeuvre-les-Nancy, F-54516, France \\ ${ }^{b}$ CNRS, CRAN, UMR 7039, France \\ ${ }^{c}$ Institut Universitaire de France
}

\begin{abstract}
This paper deals with the discrete-time switched Lur'e problem in finite domain. The aim is to provide a stabilization inside an estimate of the origin's basin of attraction, large as possible, via a suitable switching rule. The design of this switching rule is based on the min-switching policy and can be induced by sufficient conditions given by Lyapunov-Metzler inequalities. Nevertheless instead of intuitively considering a switched quadratic Lyapunov function for this approach, a suitable switched Lyapunov function including the modal nonlinearities is proposed. The assumptions required to characterize the nonlinearities are only mode-dependent sector conditions, without constraints related to the slope of the nonlinearities. An optimization problem is provided to allow the maximization of the size of the basin of attraction estimate - which may be composed of disconnected sets - under the stabilization conditions. A numerical example illustrate the efficiency of our approach and emphasize the specificities of our tools.
\end{abstract}

Keywords: Min-switching local stabilization, cone bounded nonlinearity, input saturation, basin of attraction estimate, Lyapunov-Metzler inequalities.

\footnotetext{
ฟhis work was partially supported by ANR project ArHyCo, Programme "'Systèmes Embarqués et Grandes Infrastructures"' - ARPEGE, contract number ANR-2008 SEGI 004 01-30011459 and by the European Community's Seventh Framework Programme (FP7/2007-2013) under grant agreement $\mathrm{n}^{\circ}$ 257462: HYCON2 Network of Excellence "Highly-Complex and Networked Control Systems".

Email addresses: marc.jungers@univ-lorraine.fr (Marc Jungers), Carlos-Alberto.Cavichioli-Gonzaga@univ-lorraine.fr (Carlos A. C. Gonzaga), jamal.daafouz@univ-lorraine.fr (Jamal Daafouz)
} 


\section{Introduction}

Among the widespread and rich literature of control theory for nonlinear systems [1, 2, 3], the Lur'e problem, also called the absolute stability, occupies a large place. It consists in studying the global uniform asymptotical stability of an interconnection between a linear system and a nonlinear output feedback, whatever this nonlinearity, satisfying a cone bounded sector condition [3]. Classical results for the Lur'e problem are mainly the circle criterion involving a quadratic Lyapunov function and the Popov criterion involving a Lur'e type Lyapunov function, which includes additionally an integral of the nonlinearity. When the nonlinearity satisfies only a local cone bounded sector condition, we consider the absolute stability in finite domain.

Recently such a problem has drawn renewed attention, in particular due to the refinement to cope with nonlinearities like the saturation $[4,5]$. A new Lyapunov function depending on the nonlinearity but avoiding its integral has been proposed by the authors to study the Lur'e problem in discretetime [6] and its frequency domain interpretation. This new tool offers the main advantage of not requiring additional assumption about the slope of the nonlinearity, instead of the available litterature. Moreover when considering a Lur'e problem including an input saturation, the approach using this new tool leads also to the important property that the estimate of the basin of attraction can be nonconvex and disconnected [7,6].

Switched systems are a subclass of hybrid systems and consist in the combination of a finite set of modal dynamic systems and a switching law, which indicates at each time the active mode among them [8, 9]. The issue of the stability and stabilization of switched systems, which has been clearly formalized in [10], has generated many contributions to the control theory literature (see $[11,12,13,14,15,16]$ and references therein). Mainly two frameworks should be distinguished related to the nature of the switching law. In the first case, the switching law is considered as a perturbation, that is arbitrary in the class of admissible switching laws (see [17] for more details by using a switched quadratic Lyapunov function and [18] by using a switched Lur'e type Lyapunov function). In the second case, the switching law is considered as a control input, which can be designed to stabilize the switched system. One of the most known idea is to apply a state-space partition based approach [19] or the min-switching strategy introduced in [20,8] for 
continuous-time. In this framework, sufficient conditions based on LyapunovMetzler inequalities [21, 22] are developed in association with a switched quadratic Lyapunov function [23, 24]. As suggested in [25], the intuitive use of a switched quadratic Lyapunov function can be improved by considering a switched Lur'e type Lyapunov function (see [6, 18] for its introduction). The induced state-space partition depends on the modal nonlinearities and is not reduced to a conic one. This should be regarded as an adapted property for switched nonlinear systems. To the best knowledge of the authors, there is no such a contribution related to switched Lur'e problem. More precisely, we investigate the local stabilization problem via switching rule design of a discrete-time switched system containing cone bounded sector nonlinearities and a switched (not necessarily stabilizing) feedback control law subject to the saturation. The strategy to design the switching rule is the min-switching policy. Sufficient conditions for local stabilization are provided as LyapunovMetzler inequalities, leading to a constrained optimization problem aiming at maximizing the estimate of the basin of attraction, consisting of the union of modal Lyapunov functions level sets.

The outline of the paper is as follows. Section 2 settles the switched Lur'e problem in finite domain and preliminary tools. In Section 3 the minswitching policy to stabilize the switched nonlinear system is given via a switched quadratic Lyapunov function (Proposition 1) and via a switched Lur'e type Lyapunov function (Proposition 2). These propositions introduce suitable Lyapunov-Metzler inequalities, leading to optimization problems maximizing the size of the estimate of the basin of attraction. The second approach is shown to be an extension of the first one. Section 4 is devoted to a numerical and detailed example underlining the possibilities of the main result, before concluding remarks in Section 5.

Notation: $\mathbb{R}, \mathbb{N}, \mathbb{N}^{*}$ are respectively the sets of real numbers, of natural integers and of strictly positive integers. Components of vector $x \in \mathbb{R}^{n}$ are denoted $x_{(\ell)}, \forall \ell=1, \ldots, n$. Vectors inequalities are component-wise: $x \geq 0$ means $x_{(\ell)} \geq 0$ and $x \geq y$ means $x_{(\ell)}-y_{(\ell)} \geq 0 . A_{(\ell)}\left(\operatorname{resp} . A_{i,(\ell)}\right)$ is the $\ell$-th row of matrix $A$ (resp. $A_{i}$ ). For two symmetric matrices, $A$ and $B, A>B$ means that $A-B$ is positive definite. $A^{\prime}$ denotes the transpose of matrix $A$ and for a square matrix $M, \operatorname{He}(M)=M+M^{\prime}$. $I_{n}$ (resp. $0_{n}$ ) and $0_{m \times n}$ are the $n$-order identity (null) matrix and the $m \times n$-order null matrix, respectively. The symbol $\star$ stands for symmetric block in matrices. The ellipsoidal set $\mathcal{E}(M)$ associated with $M>0$ is given by $\left\{x \in \mathbb{R}^{n} ; x^{\prime} M x \leq 1\right\}$. The set $\mathcal{M} \subset \mathbb{R}^{N \times N}$ of Metzler matri- 
ces is composed of matrices with nonnegative off-diagonal elements $\mathcal{M}=$ $\left\{\Pi \in \mathbb{R}^{N \times N}, \pi_{j i} \geq 0, \forall(i, j) \in \mathcal{I}_{N}^{2}, i \neq j\right\}$. Let us define the subclass $\mathcal{M}_{\mathrm{d}}$ of $\mathcal{M}$ such that $\mathcal{M}_{\mathrm{d}}=\left\{\Pi \in \mathcal{M}, \pi_{i i} \geq 0, \quad \sum_{\ell \in \mathcal{I}_{N}} \pi_{\ell i}=1, \forall i \in \mathcal{I}_{N}\right\}$. The convex combinations of positive definite matrices $P_{i}$ with weights being elements of the Metzler matrix $\Pi \in \mathcal{M}_{\mathrm{d}}$ is denoted $(P)_{p, i}=\sum_{\ell \in \mathcal{I}_{N}} \pi_{\ell i} P_{\ell}$.

\section{Problem statement and Preliminaries}

Consider the following discrete-time switched system composed of $N$ nonlinear modes $\left(N \in \mathbb{N}^{*}\right)$, that is the switched Lur'e system [26]:

$$
\begin{aligned}
x_{k+1} & =A_{\sigma(k)} x_{k}+F_{\sigma(k)} \varphi_{\sigma(k)}\left(C_{\sigma(k)} x_{k}\right)+B_{\sigma(t)} \operatorname{sat}\left(u_{k}\right), \quad k \in \mathbb{N}, \\
u_{k} & =K_{\sigma(k)} x_{k}+\Gamma_{\sigma(k)} \varphi_{\sigma(k)}\left(C_{\sigma(k)} x_{k}\right)
\end{aligned}
$$

where $x_{k} \in \mathbb{R}^{n}$ is the state, $u_{k} \in \mathbb{R}^{m}$ the input of the closed-loop system (1)-(2) and the functions $\varphi_{i}(\cdot)$ the nonlinearities associated with each mode $i \in \mathcal{I}_{N}=\{1, \cdots, N\}$. The notation $M_{\sigma(k)}$ means that, at each time $k, M_{\sigma(k)}$ takes its value in the set $\left\{M_{1}, \cdots, M_{N}\right\}$ indexed by $\sigma(k)$. The matrices $\left\{A_{i}\right\}_{i \in \mathcal{I}_{N}},\left\{B_{i}\right\}_{i \in \mathcal{I}_{N}},\left\{C_{i}\right\}_{i \in \mathcal{I}_{N}},\left\{F_{i}\right\}_{i \in \mathcal{I}_{N}},\left\{K_{i}\right\}_{i \in \mathcal{I}_{N}}$ and $\left\{\Gamma_{i}\right\}_{i \in \mathcal{I}_{N}}$ have appropriate dimensions: $A_{i} \in \mathbb{R}^{n \times n}, B_{i} \in \mathbb{R}^{n \times m}, F_{i} \in \mathbb{R}^{n \times p}, C_{i} \in \mathbb{R}^{p \times n}$, $K_{i} \in \mathbb{R}^{m \times n}$, and $\Gamma_{i} \in \mathbb{R}^{m \times p}$. The switching rule $\sigma: \mathbb{N} \mapsto \mathcal{I}_{N}$ indicates which mode is active at each time. In this paper, the switching rule $\sigma$ is the variable which should be designed by the controller to stabilize the closed-loop system (1)-(2). The nonlinearities $\varphi_{i}(\cdot)$ verify the following assumption.

Assumption 1. The $N$ nonlinearities $\varphi_{i}(\cdot): \mathbb{R}^{p} \rightarrow \mathbb{R}^{p}$ associated with each mode $i \in \mathcal{I}_{N}$ are assumed to satisfy their own cone bounded sector conditions and to be decentralized [3].

The statement of Assumption 1 can be rewritten abusively $\varphi_{i}(\cdot) \in\left[0_{p}, \Omega_{i}\right]$ and implies that $\varphi_{i}(0)=0$ and that there exist $N$ diagonal positive definite matrices $\Omega_{i} \in \mathbb{R}^{p \times p}$ such that independently, $\forall y \in \mathbb{R}^{p}$ and $\forall \ell=$ $1, \cdots, p, \varphi_{i, \ell)}(y)\left[\varphi_{i}(y)-\Omega_{i} y\right]_{(\ell)} \leq 0$. Hence, we have the following inequality, $\forall i \in \mathcal{I}_{N}, \forall y \in \mathbb{R}^{p}$ :

$$
\mathrm{SC}\left(\varphi_{i}(\cdot), y, \Lambda_{i}\right)=\varphi_{i}^{\prime}(y) \Lambda_{i}\left[\varphi_{i}(y)-\Omega_{i} y\right] \leq 0,
$$

for any $\Lambda_{i} \in \mathbb{R}^{p \times p}$ diagonal and positive matrices. 
The input $u_{k}$ is subject to actuation limits, and the standard saturation function is considered: $\operatorname{sat}\left(u_{k}\right)_{(\ell)}=\operatorname{sign}\left(\left(u_{k}\right)_{(\ell)}\right) \min \left(\rho_{(\ell)},\left|\left(u_{k}\right)_{(\ell)}\right|\right), \forall \ell=$ $1, \ldots, m$. The vector $0_{m}<\rho \in \mathbb{R}^{m}$ is assumed to be given.

The saturation is described as a dead-zone nonlinearity $\Psi\left(u_{k}\right)=u_{k}-$ $\operatorname{sat}\left(u_{k}\right)$. By replacing $u_{k}$ defined in (2) and using $\Psi\left(u_{k}\right)$ into (1), the closedloop model is given by

$$
x_{k+1}=A_{\mathrm{cl}, \sigma(k)} x_{k}+F_{\mathrm{cl}, \sigma(k)} \varphi_{\sigma(k)}\left(C_{\sigma(k)} x_{k}\right)-B_{\sigma(k)} \Psi\left(u_{k}\right),
$$

where $A_{\mathrm{cl}, i}=A_{i}+B_{i} K_{i}$ and $F_{\mathrm{cl}, i}=F_{i}+B_{i} \Gamma_{i}, \forall i \in \mathcal{I}_{N}$.

Let us now introduce the modal sets $\mathcal{T}_{i},\left(i \in \mathcal{I}_{N}\right)$ characterizing the deadzone $\Psi\left(u_{k}\right)$ as belonging to a generalized sector condition. For given matrices $H_{i} \in \mathbb{R}^{m \times(n+p)}, i \in \mathcal{I}_{N}$, consider $\mathcal{T}_{i}\left(H_{i}, \rho\right)=\left\{\theta \in \mathbb{R}^{n+p} ;-\rho \leq H_{i} \theta \leq \rho\right\}$.

Lemma 1. Let $i \in \mathcal{I}_{N}$ and $m \times(n+p)$-matrices $\widehat{K}_{i}=\left[K_{i} \Gamma_{i}\right]$ and $\widehat{J}_{i}=$ $\left[\begin{array}{ll}J_{1, i} & J_{2, i}\end{array}\right]$. If the vector $\widehat{x}_{k}=\left[\begin{array}{ll}x_{k}^{\prime} & \varphi_{\sigma(k)}^{\prime}\end{array}\left(C_{\sigma(k)} x_{k}\right)\right]^{\prime}$ is an element of $\mathcal{T}_{i}\left(\widehat{K}_{i}-\right.$ $\left.\widehat{J}_{i}, \rho\right)$, the nonlinearity $\Psi\left(u_{k}\right)$ satisfies the following sector condition

$$
S C_{u_{k}}=\Psi^{\prime}\left(u_{k}\right) U_{i}^{-1}\left[\Psi\left(u_{k}\right)-J_{1, i} x_{k}-J_{2, i} \varphi_{i}\left(C_{i} x_{k}\right)\right] \leq 0,
$$

with $u_{k}$ defined in (2), for any diagonal positive definite matrix $U_{i} \in \mathbb{R}^{m \times m}$

Proof 1. The proof follows the same lines of [27, Lemma 1].

Remark 2. Notice that the relation (5) is true only for the (active) mode $i \in \mathcal{I}_{N}$ on $\mathcal{T}_{i}\left(H_{i}, \rho\right)$ and is not necessarily true for all the modes.

The problem investigated in this paper is formulated as follows.

Problem 3. Consider the system (1)-(2) and Assumption 1, design a statedependent switching law

$$
\sigma(k)=g\left(x_{k}\right)
$$

which makes the origin of system (1)-(2) locally asymptotically stable.

In order to cope with the problem 3, we define the intuitive switched quadratic function $Q$ and the Lur'e type Lyapunov function $V$, introduced in [18]:

$$
Q:\left\{\begin{array}{lll}
\mathcal{I}_{N} \times \mathbb{R}^{n} & \longrightarrow & \mathbb{R}, \\
(i, x) & \longmapsto & x^{\prime} \tilde{P}_{i} x,
\end{array}\right.
$$




$$
V:\left\{\begin{array}{lll}
\mathcal{I}_{N} \times \mathbb{R}^{n} \times \mathbb{R}^{p} & \longrightarrow \mathbb{R} \\
\left(i, x, \varphi_{i}\left(C_{i} x\right)\right) & \longmapsto x^{\prime} P_{i} x+2\left(\varphi_{i}\left(C_{i} x\right)\right)^{\prime} \Delta_{i} \Omega_{i} C_{i} x
\end{array}\right.
$$

where $\tilde{P}_{i} \in \mathbb{R}^{n \times n}$ and $P_{i} \in \mathbb{R}^{n \times n}\left(i \in \mathcal{I}_{N}\right)$ are symmetric positive definite matrices and $\Delta_{i} \in \mathbb{R}^{p \times p}$ are diagonal positive semidefinite matrices.

Remark 4. It is noteworthy that the switched quadratic Lyapunov function $Q$ is a particular case of the function $V$, which is obtained by fixing $\Delta_{i}=0_{p}$, $\forall i \in \mathcal{I}_{N}$ and identifying $P_{i}=\tilde{P}_{i}$ in the definitions (7) and (8).

In view of the min-switching based control, we define the functions

$V_{\text {min }}:\left\{\begin{array}{lll}\mathbb{R}^{n} & \longrightarrow \mathbb{R}, \\ x & \longmapsto & \min _{i \in \mathcal{I}_{N}} V\left(i ; x ; \varphi_{i}\left(C_{i} x\right)\right)\end{array} ; \quad Q_{\min }:\left\{\begin{array}{lll}\mathbb{R}^{n} & \longrightarrow \mathbb{R}, \\ x & \longmapsto & \min _{i \in \mathcal{I}_{N}} Q(i ; x) .\end{array}\right.\right.$

and their level sets related to the unitary level by

$$
L_{V_{\min }}(1)=\left\{x \in \mathbb{R}^{n} ; V_{\min }(x) \leq 1\right\} ; \quad L_{Q_{\min }}(1)=\left\{x \in \mathbb{R}^{n} ; Q_{\min }(x) \leq 1\right\} .
$$

The level sets $L_{V_{\text {min }}}(1)$ and $L_{Q_{\min }}(1)$ can be reformulated into the following unions [23]:

$$
L_{V_{\min }}(1)=\bigcup_{j \in \mathcal{I}_{N}}\left\{x \in \mathbb{R}^{n} ; V\left(j ; x ; \varphi_{j}\left(C_{j} x\right)\right) \leq 1\right\} ; \quad L_{Q_{\min }}(1)=\bigcup_{j \in \mathcal{I}_{N}} \mathcal{E}\left(\tilde{P}_{j}\right)
$$

Sufficient conditions ensuring that $V_{\min }\left(\right.$ or $\left.Q_{\min }\right)$ is decreasing along the trajectory will be provided in the following section.

\section{Min-switching based stabilization}

The following propositions are related to the solution of the problem 3 , respectively with Lyapunov functions $V_{\min }$ and $Q_{\min }$.

Proposition 1. Consider the closed-loop switched system (4). Assume there exists a matrix $\Pi \in \mathcal{M}_{d}$; symmetric positive definite matrices $\tilde{P}_{i} \in \mathbb{R}^{n \times n}$, diagonal positive definite matrices $\tilde{Z}_{i}, \tilde{T}_{i} \in \mathbb{R}^{p \times p}$, diagonal positive definite matrices $\tilde{U}_{i} \in \mathbb{R}^{m \times m}$, matrices $\tilde{J}_{1, i} \in \mathbb{R}^{m \times n}, \tilde{J}_{2, i} \in \mathbb{R}^{m \times p}\left(i \in \mathcal{I}_{N}\right)$, such that 
the Lyapunov-Metzler inequalities are satisfied $\forall i \in \mathcal{I}_{N}$ :

$$
\left[\begin{array}{c}
A_{c l, i}^{\prime} \\
F_{c l, i}^{\prime} \\
-B_{i}^{\prime}
\end{array}\right](\tilde{P})_{p, i}\left[\begin{array}{c}
A_{c l, i}^{\prime} \\
F_{c l, i}^{\prime} \\
-B_{i}^{\prime}
\end{array}\right]^{\prime}-\left[\begin{array}{ccc}
\tilde{P}_{i} & \star & \star \\
-\tilde{T}_{i} \Omega_{i} C_{i} & 2 \tilde{T}_{i} & \star \\
-\tilde{U}_{i}^{-1} \tilde{J}_{1, i} & -\tilde{U}_{i}^{-1} \tilde{J}_{2, i} & 2 \tilde{U}_{i}^{-1}
\end{array}\right]<0_{n+m+p}
$$

and LMIs $\forall(i, \ell) \in \mathcal{I}_{N} \times\{1, \cdots, m\}$

$$
\left[\begin{array}{ccc}
\tilde{P}_{i} & \star & \star \\
-\tilde{Z}_{i} \Omega_{i} C_{i} & 2 \tilde{Z}_{i} & \star \\
\left(K_{i}-\tilde{J}_{1, i}\right)_{(\ell)} & \left(\Gamma_{i}-\tilde{J}_{2, i}\right)_{(\ell)} & \rho_{(\ell)}^{2}
\end{array}\right]>0_{n+p+1},
$$

then the switching law

$$
\sigma(k) \in \arg \min _{i \in \mathcal{I}_{N}} Q\left(i, x_{k}\right)
$$

locally asymptotically stabilizes the system (4) on $L_{Q_{m i n}}(1)$.

Proposition 2. Consider the closed-loop switched system (4). Assume there exists a matrix $\Pi \in \mathcal{M}_{d}$; symmetric positive definite matrices $P_{i} \in \mathbb{R}^{n \times n}$, diagonal positive definite matrices $Z_{i}, T_{i} \in \mathbb{R}^{p \times p}$, diagonal positive semidefinite matrices $\Delta_{i}, W_{i} \in \mathbb{R}^{p \times p}$, diagonal positive definite matrices $U_{i} \in \mathbb{R}^{m \times m}$, matrices $J_{1, i} \in \mathbb{R}^{m \times n}, J_{2, i} \in \mathbb{R}^{m \times p}\left(i \in \mathcal{I}_{N}\right)$, such that the Lyapunov-Metzler inequalities are satisfied $\forall i \in \mathcal{I}_{N}$ :

$$
\begin{array}{r}
\mathbb{A}_{i}^{\prime}(P)_{p, i} \mathbb{A}_{i}+\operatorname{He}\left(\mathbb{A}_{i}^{\prime}\left(C^{\prime} \Omega \Delta \mathbb{E}\right)_{p, i}\right)-\sum_{q \in \mathcal{I}_{N}}\left(2 \mathbb{E}_{q}^{\prime} W_{q} \mathbb{E}_{q}-\operatorname{He}\left(\mathbb{E}_{q}^{\prime} W_{q} \Omega_{q} C_{q} \mathbb{A}_{i}\right)\right) \\
-\left[\begin{array}{cccc}
P_{i} & \star & \star & \star \\
\left(\Delta_{i}-T_{i}\right) \Omega_{i} C_{i} & 2 T_{i} & \star & \star \\
-U_{i}^{-1} J_{1, i} & -U_{i}^{-1} J_{2, i} & 2 U_{i}^{-1} & \star \\
0_{N p \times n} & 0_{N p \times p} & 0_{N p \times m} & 0_{N p}
\end{array}\right]<0_{\hat{n}}
\end{array}
$$

and LMIs $\forall(i, \ell) \in \mathcal{I}_{N} \times\{1, \cdots, m\}$

$$
\left[\begin{array}{ccc}
P_{i} & \star & \star \\
\left(\Delta_{i}-Z_{i}\right) \Omega_{i} C_{i} & 2 Z_{i} & \star \\
\left(K_{i}-J_{1, i}\right)_{(\ell)} & \left(\Gamma_{i}-J_{2, i}\right)_{(\ell)} & \rho_{(\ell)}^{2}
\end{array}\right]>0_{n+p+1}
$$


where $\forall i \in \mathcal{I}_{N}$,

$$
\begin{gathered}
\mathbb{A}_{i}=\left[\begin{array}{llll}
A_{c l, i} & F_{c l, i} & -B_{i} & 0_{n \times N p}
\end{array}\right], \\
\mathbb{E}_{i}=\left[\begin{array}{lll}
0_{p \times(n+p+m+i p)} & I_{p} & 0_{p \times(N-i-1) p}
\end{array}\right],
\end{gathered}
$$

and with $\hat{n}=n+m+(N+1) p$, then the switching law

$$
\sigma(k) \in \arg \min _{i \in \mathcal{I}_{N}} V\left(i, x_{k}, \varphi_{i}\left(C_{i} x_{k}\right)\right)
$$

locally asymptotically stabilizes the system (4) on $L_{V_{m i n}}(1)$.

Proof 5. The proofs of propositions 1 and 2 are similar and are based on the different extended state vectors $\tilde{z}_{k}$ and $z_{k}$, which contains $\tilde{z}_{k}$ and all the nonlinearities at time $k+1$. They are defined by

$$
\begin{gathered}
\tilde{z}_{k}=\left(\begin{array}{lllll}
x_{k}^{\prime} & \varphi_{i}^{\prime}\left(C_{i} x_{k}\right) & \Psi^{\prime}\left(u_{k}\right)
\end{array}\right)^{\prime} \in \mathbb{R}^{n+p+m} ; \\
z_{k}=\left(\begin{array}{llllll}
x_{k}^{\prime} & \varphi_{i}^{\prime}\left(C_{i} x_{k}\right) & \Psi^{\prime}\left(u_{k}\right) & \varphi_{1}^{\prime}\left(C_{1} x_{k+1}\right) & \cdots & \varphi_{N}^{\prime}\left(C_{N} x_{k+1}\right)
\end{array}\right)^{\prime} \in \mathbb{R}^{\hat{n}} .
\end{gathered}
$$

For the sake of clarity, only proposition 2 will be proven. The proof consists in ensuring that under the assumptions of proposition 2, the candidate Lyapunov function $V_{\text {min }}$ is decreasing along the trajectory on $L_{V_{\min }}(1)$. Due to the structure of the extended state $z_{k}, x_{k}=0$ is equivalent to $z_{k}=0$. The notations (17)-(18) yield that $x_{k+1}=\mathbb{A}_{\sigma(k)} z_{k}$ and $\varphi_{j}\left(C_{j} x_{k+1}\right)=\mathbb{E}_{j} z_{k}$. The sector conditions at time $k+1$, related to the mode $q \in \mathcal{I}_{N}$, with $W_{q}$ diagonal positive semidefinite matrices write $-2 \varphi_{q}^{\prime}\left(C_{q} x_{k+1}\right) W_{q}\left[\varphi_{q}\left(C_{q} x_{k+1}\right)-\right.$ $\left.\Omega_{q} C_{q} x_{k+1}\right] \geq 0$, which is equivalent to

$$
-z_{k}^{\prime}\left(2 \mathbb{E}_{q}^{\prime} W_{q} \mathbb{E}_{q}-\operatorname{He}\left(\mathbb{E}_{q}^{\prime} W_{q} \Omega_{q} C_{q} \mathbb{A}_{i}\right)\right) z_{k} \geq 0 .
$$

By evaluating $V_{\min }\left(x_{k+1}\right)$ as

$$
\begin{aligned}
V_{\text {min }}\left(x_{k+1}\right) & =\min _{j \in \mathcal{I}_{N}} V\left(j, x_{k+1}, \varphi_{j}\left(C_{j} x_{k+1}\right)\right), \\
& =\min _{j \in \mathcal{I}_{N}} z_{k}^{\prime}\left(\mathbb{A}_{i}^{\prime} P_{j} \mathbb{A}_{i}+\operatorname{He}\left(\mathbb{A}_{i}^{\prime} C_{j}^{\prime} \Omega_{j} \Delta_{j} \mathbb{E}_{j}\right)\right) z_{k},
\end{aligned}
$$

let us upper bound $V_{\min }\left(x_{k+1}\right)$ by adding the sector conditions (22) for all $q \in \mathcal{I}_{N}$ :

$$
\begin{aligned}
V_{\text {min }}\left(x_{k+1}\right) \leq \min _{j \in \mathcal{I}_{N}} z_{k}^{\prime}\left(\mathbb{A}_{i}^{\prime} P_{j} \mathbb{A}_{i}+\operatorname{He}\left(\mathbb{A}_{i}^{\prime} C_{j}^{\prime} \Omega_{j} \Delta_{j} \mathbb{E}_{j}\right)\right. \\
\left.-\sum_{q \in \mathcal{I}_{N}}\left(2 \mathbb{E}_{q}^{\prime} W_{q} \mathbb{E}_{q}-\operatorname{He}\left(\mathbb{E}_{q}^{\prime} W_{q} \Omega_{q} C_{q} \mathbb{A}_{i}\right)\right)\right) z_{k} .
\end{aligned}
$$


The upper bound (24) involves a minimum of a finite number of scalars. It yields, with $\Theta_{N}$ the unit simplex of dimension $N$,

$$
\begin{aligned}
V_{\min }\left(x_{k+1}\right) \leq \min _{\gamma \in \Theta_{N}} \sum_{j \in \mathcal{I}_{N}} \gamma_{(j)} z_{k}^{\prime}\left(\mathbb{A}_{i}^{\prime} P_{j} \mathbb{A}_{i}+\operatorname{He}\left(\mathbb{A}_{i}^{\prime} C_{j}^{\prime} \Omega_{j} \Delta_{j} \mathbb{E}_{j}\right)\right. \\
\left.-\sum_{q \in \mathcal{I}_{N}}\left(2 \mathbb{E}_{q}^{\prime} W_{q} \mathbb{E}_{q}-\operatorname{He}\left(\mathbb{E}_{q}^{\prime} W_{q} \Omega_{q} C_{q} \mathbb{A}_{i}\right)\right)\right) z_{k} .
\end{aligned}
$$

Each column of the matrix $\Pi \in \mathcal{M}_{d}$ belonging to $\Theta_{N}$ by definition, we obtain

$$
\begin{aligned}
V_{\text {min }}\left(x_{k+1}\right) \leq \sum_{j \in \mathcal{I}_{N}} \pi_{j i} z_{k}^{\prime}\left(\mathbb{A}_{i}^{\prime} P_{j} \mathbb{A}_{i}+\operatorname{He}\left(\mathbb{A}_{i}^{\prime} C_{j}^{\prime} \Omega_{j} \Delta_{j} \mathbb{E}_{j}\right)\right. \\
\left.-\sum_{q \in \mathcal{I}_{N}}\left(2 \mathbb{E}_{q}^{\prime} W_{q} \mathbb{E}_{q}-\operatorname{He}\left(\mathbb{E}_{q}^{\prime} W_{q} \Omega_{q} C_{q} \mathbb{A}_{i}\right)\right)\right) z_{k},
\end{aligned}
$$

that is

$$
\begin{aligned}
V_{\text {min }}\left(x_{k+1}\right) \leq z_{k}^{\prime}\left(\mathbb{A}_{i}^{\prime}(P)_{p, i}\right. & \mathbb{A}_{i}+\operatorname{He}\left(\mathbb{A}_{i}^{\prime}\left(C^{\prime} \Omega \Delta \mathbb{E}\right)_{p, i}\right) \\
& \left.-\sum_{q \in \mathcal{I}_{N}}\left(2 \mathbb{E}_{q}^{\prime} W_{q} \mathbb{E}_{q}-\operatorname{He}\left(\mathbb{E}_{q}^{\prime} W_{q} \Omega_{q} C_{q} \mathbb{A}_{i}\right)\right)\right) z_{k} .
\end{aligned}
$$

Thanks to inequality (16), we have

$$
V_{\min }\left(x_{k+1}\right) \leq z_{k}^{\prime}\left[\begin{array}{cccc}
P_{i} & \star & \star & \star \\
\left(\Delta_{i}-T_{i}\right) \Omega_{i} C_{i} & 2 T_{i} & \star & \star \\
-U_{i}^{-1} J_{1, i} & -U_{i}^{-1} J_{2, i} & 2 U_{i}^{-1} & \star \\
0_{N p \times n} & 0_{N p \times p} & 0_{N p \times m} & 0_{N p}
\end{array}\right] z_{k} .
$$

Identifying $i=\sigma(k) \in \arg \min _{j \in \mathcal{I}_{N}} V\left(j ; x_{k} ; \varphi_{j}\left(C_{j} x_{k}\right)\right)$, one gets

$$
\begin{aligned}
V_{\min }\left(x_{k+1}\right) \leq V_{\min }\left(x_{k}\right) & +2 \varphi_{i}^{\prime}\left(C_{i} x_{k}\right) T_{i}\left[\varphi_{i}\left(C_{i} x_{k}\right)-\Omega_{i} C_{i} x_{k}\right] \\
& +2 \Psi^{\prime}\left(u_{k}\right) U_{i}^{-1}\left[\Psi\left(u_{k}\right)-J_{1, i} x_{k}-J_{2, i} \varphi_{i}\left(C_{i} x_{k}\right)\right],
\end{aligned}
$$

which implies

$$
V_{\min }\left(x_{k+1}\right)-V_{\min }\left(x_{k}\right) \leq 2 \Psi^{\prime}\left(u_{k}\right) U_{i}^{-1}\left[\Psi\left(u_{k}\right)-J_{1, i} x_{k}-J_{2, i} \varphi_{i}\left(C_{i} x_{k}\right)\right] .
$$


Due to Inequality (16), we have by applying a Schur Complement,

$\left[\begin{array}{cc}P_{i} & \star \\ \left(\Delta_{i}-Z_{i}\right) \Omega_{i} C_{i} & 2 Z_{i}\end{array}\right]>\frac{1}{\rho_{(\ell)}^{2}}\left(\left[\begin{array}{ll}K_{i} & \Gamma_{i}\end{array}\right]-\left[\begin{array}{ll}J_{1, i} & J_{2, i}\end{array}\right]\right)_{(\ell)}^{\prime}\left(\left[\begin{array}{ll}K_{i} & \Gamma_{i}\end{array}\right]-\left[\begin{array}{ll}J_{1, i} & J_{2, i}\end{array}\right]\right)($

By multiplying Inequality (31) on the right by $\widehat{x}_{k}=\left[\begin{array}{ll}x_{k}^{\prime} & \varphi_{i}^{\prime}\left(C_{i} x_{k}\right)\end{array}\right]^{\prime}$ and on the left by its transpose, one has the following inequality

$$
\begin{aligned}
V\left(i, x_{k}, \varphi_{i}\left(C_{i} x_{k}\right)\right) & \geq V\left(i, x_{k}, \varphi_{i}\left(C_{i} x_{k}\right)\right)+2 \varphi_{i}^{\prime}\left(C_{i} x_{k}\right) Z_{i}\left[\varphi_{i}\left(C_{i} x_{k}\right)-\Omega_{i} C_{i} x_{k}\right] \\
& \geq \frac{1}{\rho_{(\ell)}^{2}}\left\|\left(\left[K_{i} \Gamma_{i}\right]-\left[\begin{array}{ll}
J_{1, i} & J_{2, i}
\end{array}\right]\right)_{(\ell)} \widehat{x}_{k}\right\|^{2}
\end{aligned}
$$

For $x_{k} \in L_{V_{\text {min }}}(1)$, the mode which will be activated by the switching law $\sigma(k)=g\left(x_{k}\right)$ given by (19) verifies $V\left(\sigma(k), x_{k}, \varphi_{\sigma(k)}\left(C_{\sigma(k)} x_{k}\right)\right) \leq 1$. Notice that this relation is ensured for the modes belonging to $\operatorname{argmin}_{j \in \mathcal{I}_{N}} V\left(j, x_{k}, \varphi_{j}\left(C_{j} x_{k}\right)\right)$ and not necessarily for the other ones. Due to inequality (32), it induces that the sector condition for the deadzone $\Psi\left(u_{k}\right)$ is verified for the active mode $\sigma(k)$. The inequality (30) can be simplified as

$$
V_{\min }\left(x_{k+1}\right)-V_{\min }\left(x_{k}\right)<0, \quad \forall x_{k} \neq 0,
$$

which concludes the proof.

Remark 6. It is noteworthy that if the inequalities (12) and (13) are feasible, this is also the case of inequalities (15) and (16), by setting $P_{i}=\tilde{P}_{i}$, $T_{i}=\tilde{T}_{i}, U_{i}=\tilde{U}_{i}, Z_{i}=\tilde{Z}_{i}, J_{1, i}=\tilde{J}_{1, i}, J_{2, i}=\tilde{J}_{2, i}, \Delta_{i}=0_{p}$ and $W_{i}=0_{p}$.

The basin of attraction of the origin may be estimated by $L_{V_{\min }}(1)$, thanks to the proposition 2. The solution of the problem 3 is given by the following optimization problems related to the both considered Lyapunov functions. $R_{i} \in \mathbb{R}^{p \times p}$ are diagonal positive definite matrices and $\mu_{i}, \tilde{\mu}_{i}$ are positive definite scalars $\left(i \in \mathcal{I}_{N}\right)$.

\section{Optimization problem 1.}

$$
\min _{\Pi, \tilde{P}_{i}, \tilde{U}_{i}, \tilde{Z}_{i}, \tilde{T}_{i}, \tilde{J}_{1, i}, \tilde{J}_{2, i}, \tilde{\mu}_{i}} \sum_{j \in \mathcal{I}_{N}} \tilde{\mu}_{j}
$$

under the constraints (12)-(13) and $\tilde{\mu}_{i} I_{n}>\tilde{P}_{i}$. 


\section{Optimization problem 2.}

$$
\min _{\Pi, P_{i}, U_{i}, Q_{i}, T_{i}, W_{i}, R_{i}, \Delta_{i}, J_{1, i}, J_{2, i}, \mu_{i}} \sum_{j \in \mathcal{I}_{N}} \mu_{j}
$$

under the constraints (15)-(16) and

$$
\left[\begin{array}{cc}
\mu_{i} I_{n}-P_{i} & \star \\
-\left(\Delta_{i}+R_{i}\right) \Omega_{i} C_{i} & 2 R_{i}
\end{array}\right]>0_{n+p}
$$

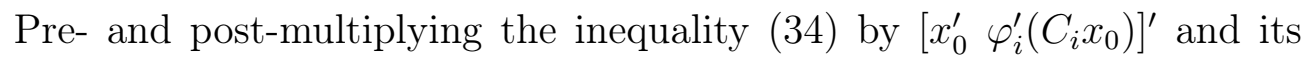
transpose leads to

$$
\begin{aligned}
\mu_{i} x_{0}^{\prime} x_{0} & \geq V\left(i, x_{0}, \varphi_{i}\left(C_{i} x_{0}\right)\right)-2 \varphi_{i}^{\prime}\left(C_{i} x_{0}\right) R_{i}\left[\varphi_{i}\left(C_{i} x_{0}\right)-R_{i} \Omega_{i} C_{i} x_{0}\right] \\
& \geq V\left(i, x_{0}, \varphi_{i}\left(C_{i} x_{0}\right)\right)
\end{aligned}
$$

which can be interpreted as the inclusion

$$
\mathcal{E}\left(\mu_{i} I_{n}\right) \subset\left\{x \in \mathbb{R}^{n} ; V\left(i ; x ; \varphi_{i}\left(C_{i} x\right)\right) \leq 1\right\} \subset L_{V_{\min }}(1) .
$$

Minimizing the cost function $\sum_{j \in \mathcal{I}_{N}} \mu_{j}$ in the optimization problem 2, leads to increase the size of the balls $\mathcal{E}\left(\mu_{i} I_{n}\right)$ and thus to increase the size of the estimate of the basin of attraction of the origin $L_{V_{\min }}(1)$.

Remark 7. The level set $L_{V_{\text {min }}}(1)$, the estimate of the basin of attraction of the origin, being a function of the modal nonlinearities may be the union of non-convex and disconnected sets. The notion of the size of such a set is difficult to define. Several definitions may occur. We made the choice to consider as a definition, the size of the biggest ball inside $L_{V_{\min }}(1)$. Other choices may be possible leading to a modification of the criterion to be minimized in the optimization problem.

Remark 8. It should be emphasized that necessary conditions of the feasibility of inequalities (12) and (15) are that $\pi_{i i}^{\frac{1}{2}} A_{c l, i}$ and $\pi_{i i}^{\frac{1}{2}}\left(A_{c l, i}+F_{c l, i} \Omega_{i} C_{i}\right)$ are Schur, $\forall i \in \mathcal{I}_{N}$. This can be shown similarly as in [28, 25], by considering the particular cases $\varphi_{i}\left(C_{i} x\right)=\Omega_{i} C_{i} x$ and $\varphi_{i}\left(C_{i} x\right)=0$. Nevertheless, $A_{c l, i}$ and $\left(A_{c l, i}+F_{c l, i} \Omega_{i} C_{i}\right)$ are not required to be Schur. 
The inequalities (12) and (15) are Bilinear Matrix Inequalities (BMIs), if the matrix $\Pi \in \mathcal{M}_{\mathrm{d}}$ (and $U_{i}$ ) is a variable, but a LMIs if $\Pi$ and $U_{i}$ are fixed. The other inequalities in proposition 2 are LMIs. Sufficient conditions are proposed in $[28,29]$ by considering a subclass of Metzler matrices depending only on a single scalar parameter and a line search procedure. In order to emphasize our main result about the improvement of the class of switched Lyapunov functions, we made the choice to impose in the example $\Pi=$ $\left[\begin{array}{cc}\alpha & 1-\alpha \\ 1-\alpha & \alpha\end{array}\right]$, with a fixed $\alpha$.

\section{Illustration}

Let us consider the following example to illustrate our results. The system (4) is defined by $N=2, n=2, m=p=1, \rho=5$ and

$$
\begin{gathered}
A_{1}=\left[\begin{array}{cc}
1.4 & 0.4 \\
0.2 & 1
\end{array}\right] ; A_{2}=\left[\begin{array}{cc}
1.1 & 0.6 \\
0.3 & 1.5
\end{array}\right] ; B_{1}=\left[\begin{array}{c}
0.5 \\
0.5
\end{array}\right] ; B_{2}=\left[\begin{array}{l}
0.7 \\
0.5
\end{array}\right] ; \\
F_{1}=\left[\begin{array}{c}
1 \\
1.2
\end{array}\right] ; F_{2}=\left[\begin{array}{c}
1.2 \\
1
\end{array}\right] ; C_{1}=\left[\begin{array}{cc}
0.9 & 0.5
\end{array}\right] ; C_{2}=\left[\begin{array}{cc}
1 & 0.7
\end{array}\right] ; \\
K_{1}=[-0.7168-1.0136] ; K_{2}=\left[\begin{array}{ll}
-1.2581-0.7326
\end{array}\right] ; \\
\Gamma_{1}=[-1.2923] ; \Gamma_{2}=[-1.4650] ; \Omega_{1}=0.7 ; \Omega_{2}=0.5 ;
\end{gathered}
$$

and the nonlinearities

$$
\varphi_{1}(y)=0.5 \Omega_{1} y(1+\cos (20 y)) ; \quad \varphi_{2}(y)=0.5 \Omega_{2} y(1-\sin (25 y)) .
$$

In addition, we impose $U_{1}=U_{2}=0.1$ and $\alpha=0.5$. The matrices $A_{\mathrm{cl}, i}$ and $A_{\mathrm{cl}, i}+F_{\mathrm{cl}, i} \Omega_{i} C_{i}$ are not Schur, $\left(\forall i \in \mathcal{I}_{N}\right)$, instead of $\alpha^{\frac{1}{2}} A_{\mathrm{cl}, i}$ and

$\alpha^{\frac{1}{2}}\left(A_{\mathrm{cl}, i}+F_{\mathrm{cl}, i} \Omega_{i} C_{i}\right)$. The optimization problems given in Section 3 lead to solutions described as follows.

The estimate $L_{V_{\min }}(1)$ and $L_{Q_{\min }}(1)$ of the basin of attraction of the origin are respectively plotted in Figure 1 and 2 . For this example, $L_{V_{\min }}(1)$, instead of $L_{Q_{\text {min }}}(1)$, is the union of distinct and disconnected sets, due to the presence of the nonlinearities in the Lyapunov function $V_{\min }$. Moreover, $L_{Q_{\min }}(1)$ is included in the subset of $L_{V_{\min }}(1)$ containing the origin. The switched Lur'e type Lyapunov function leads to better results than the switched quadratic one. Inside the set $L_{V_{\min }}(1)$, a state-space partition is emphasized in Figure 1, 
by the sets $\mathcal{S}_{i}$, defined by $\mathcal{S}_{i}=\left\{x \in \mathbb{R}^{n}, V_{\min }(x)=V\left(i, x, \varphi_{i}\left(C_{i} x\right)\right)\right\}$ and related to the possible activation of mode $i \in \mathcal{I}_{N}$, in order to design the switching rule $\sigma(k)=g\left(x_{k}\right)$ given by (19). It is noteworthy that the bounds of the activation areas, that is $\left\{x \in \mathbb{R}^{n}, V\left(1, x, \varphi_{1}\left(C_{1} x\right)\right)=V\left(2, x, \varphi_{2}\left(C_{2} x\right)\right)\right\}$, are not composed of straight lines, but curves which are characteristic of the presence of the nonlinearities in the function $V$ given by (8). The partition associated with the switched quadratic Lyapunov function consists of straight lines, solutions of $\left\{x \in \mathbb{R}^{n}, Q(1, x)=Q(2, x)\right\}$ inside $L_{Q_{\min }}(1)$ in Figure 2.

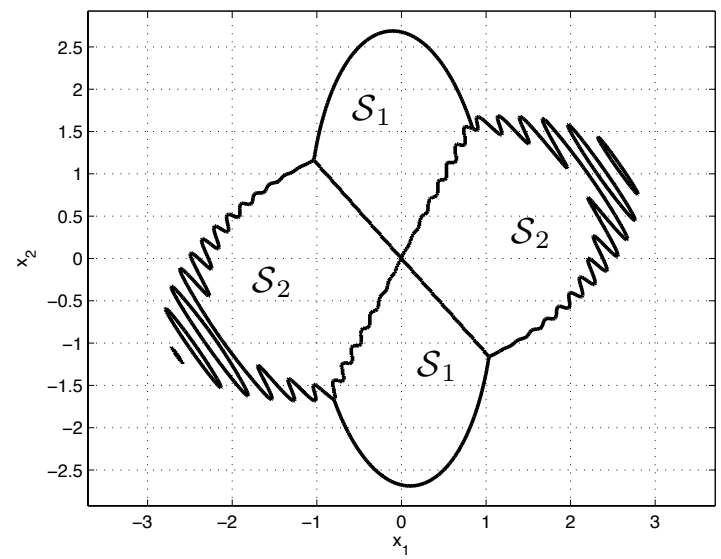

Figure 1: $L_{V_{\min }}(1)$ and the related partition $\left(\mathcal{S}_{1} ; \mathcal{S}_{2}\right)$.

In Figure 3, a state-trajectory is emphasized in the state-space. The trajectory starts from the initial condition $x_{0}=(2.09201 .4083)^{\prime}$ belonging to a subset of $L_{V_{\min }}(1)$ which does not contain the origin.

Because the main proposition provides sufficient stability conditions, a question about the gap between the basin of attraction $\mathcal{B}_{0}$ and the disconnected estimate set $L_{V_{\min }}(1)$ may arise. Hence, we have analyzed the trajectories for initial conditions located on a grid in the phase portrait. If the trajectory is unstable by applying the switching law given by (19), the initial state is marked in Figure 4. It is emphasized in Figure 4, that the estimate $L_{V_{\min }}(1)$ is close to initial states of unstable trajectories. Moreover, the shape of the set $L_{V_{\min }}(1)$ are emphasized to be suitable to the repartition of initial conditions of unstable trajectories. 


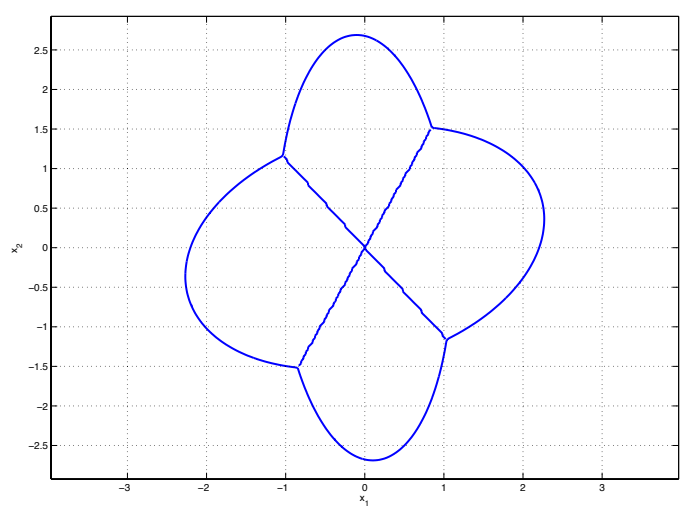

Figure 2: $L_{Q_{\min }}(1)$ and the related partition.

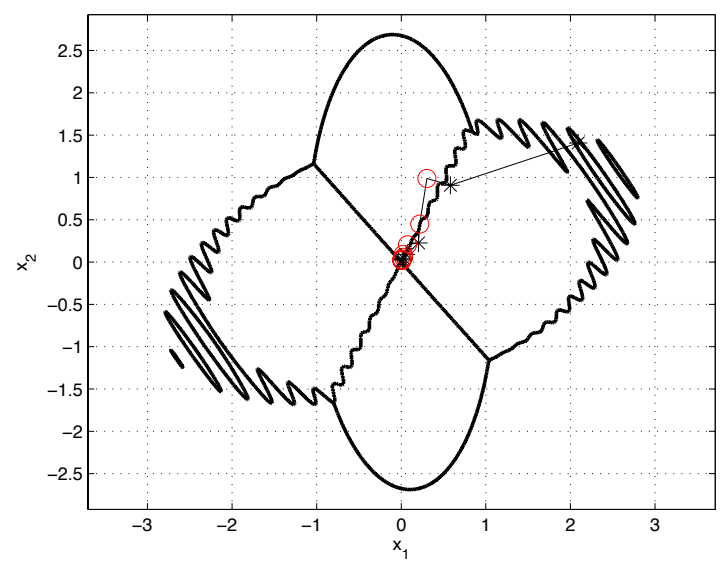

Figure 3: The set $L_{V_{\min }}(1)$ and the switching law state-space partition are depicted in black lines, in addition of the trajectory starting from $x_{0}=(2.09201 .4083)^{\prime}$. At each state $x_{k}$, a red circle (resp. a black star) denotes that the mode 1 (resp. 2) is selected.

\section{Conclusion}

Local stabilization of a discrete-time switched system including modal nonlinearities and saturated feedbacks via the design of the switching law has been investigated in this note. The synthesis of the switching law is based on a min-switching policy related to switched Lyapunov functions taking into 


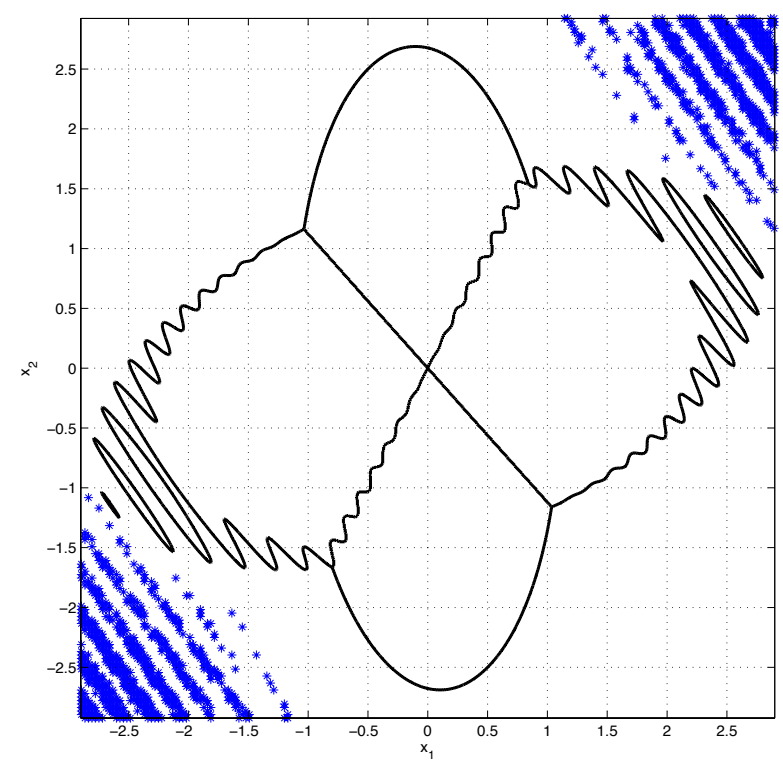

Figure 4: $L_{V_{\min }}(1)$ and initial conditions leading to unstable trajectories by applying the switching law $\sigma(k)$ defined by (19).

account the modal nonlinearities and leads to a state-space partition of the basin of attraction estimate. Optimization problems, involving LyapunovMetzler inequalites have been provided to look for the maximal size of this estimate under local stabilizing conditions. A numerical example allows the interpretation of our results and to emphasize the suitability of the switched Lur'e type Lyapunov functions with respect to the quadratic ones.

\section{References}

[1] J.-J. E. Slotine, W. Li, Applied nonlinear control, Prentice-Hall International Editions, 1991.

[2] M. Vidyasagar, Nonlinear Systems Theory, Prentice-Hall International Editions, 1993.

[3] H. K. Khalil, Nonlinear Systems - Third Edition, Prentice Hall, 2002.

[4] T. Hu, Z. Lin, Control systems with actuator saturation. Analysis and design, Birkhäuser, 2001. 
[5] S. Tarbouriech, G. Garcia, J. M. Gomes da Silva Jr., I. Queinnec, Stability and Stabilization of Linear Systems with Saturating Actuators, Springer-Verlag London, 2011.

[6] C. A. C. Gonzaga, M. Jungers, J. Daafouz, Stability analysis of discretetime Lur'e systems, Automatica 48 (2012) 2277-2283.

[7] C. A. C. Gonzaga, M. Jungers, J. Daafouz, E. B. Castelan, Stabilization of discrete-time nonlinear systems subject to input saturations: a new Lyapunov function class, in: IFAC World Congress, Milan, Italy, pp. 3403-3408.

[8] D. Liberzon, Switching in Systems and Control, volume in series Systems and Control: Foundations and Applications., Birkhäuser, Boston, MA, 2003.

[9] R. Decarlo, M. Branicky, S. Pettersson, B. Lennartson, Perspective and results on the stability and stabilization of hybrid systems., Proceedings of the IEEE: Special issue on hybrid systems 88 (2000) 1069-1082.

[10] D. Liberzon, A. Morse, Basic problems in stability and design of switched systems, IEEE Control Systems Magazine 19 (1999) 59-70.

[11] S. Pettersson, B. Lennartson, LMI for stability and robustness for hybrid systems, in: Proceedings of the American Control Conference, pp. 17141718.

[12] J. Daafouz, P. Riedinger, C. Iung, Stability analysis and control synthesis for switched systems : A switched Lyapunov function approach, IEEE Transactions on Automatic Control 47 (2002) 1883-1887.

[13] V. D. Blondel, Y. Nesterov, Computationally efficient approximations of the joint spectral radius, SIAM Journal of Matrix Analysis 27 (2005) $256-272$.

[14] J. W. Lee, G. E. Dullerud, Uniformly stabilizing sets of switching sequences for switched linear systems, IEEE Transactions on Automatic Control 52 (2007) 868-874.

[15] R. M. Jungers, The joint spectral radius. Theory and applications, Springer, 2009. 
[16] H. Lin, P. J. Antsaklis, Stability and stabilizability of switched linear systems: A survey of recent results, IEEE Transactions on Automatic Control 54 (2009) 308-322.

[17] M. Jungers, E. B. Castelan, S. Tarbouriech, J. Daafouz, Finite $\mathcal{L}_{2^{-}}$ induced gain and $\lambda$-contractivity of discrete-time switching systems including modal nonlinearities and saturating actuator, Nonlinear Analysis: Hybrid Systems 5 (2011) 289-300.

[18] C. A. C. Gonzaga, M. Jungers, J. Daafouz, Stability analysis and stabilization of switched nonlinear systems, International Journal of Control 85 (2012) 822-829.

[19] M. Johansson, A. Rantzer, Computation of piecewise quadratic Lyapunov functions for hybrid systems, IEEE Transactions on Automatic Control 43 (1998) 555-559.

[20] M. Wicks, R. DeCarlo, Solution of coupled Lyapunov equations for the stabilization of multimodal linear systems, in: Proceedings of the American Control Conference, pp. 1709-1713.

[21] H. Abou-Kandil, G. Freiling, V. Ionescu, G. Jank, Matrix Riccati Equations in Control and Systems Theory, Birkhäuser, 2003.

[22] H. Abou-Kandil, G. Freiling, G. Jank, Solution and asymptotic behavior of coupled Riccati equations in jump linear systems, IEEE Transactions on Automatic Control AC-39 (1994) 1631-1636.

[23] T. Hu, Z. Lin, Composite quadratic Lyapunov functions for constrained control systems, IEEE Transactions on Automatic Control 48 (2003) 440-450.

[24] T. Hu, Switching law construction for discrete-time systems via composite quadratic functions, in: Proceedings of the 2007 American Control Conference, New York City, USA, pp. 675-680.

[25] M. Jungers, C. A. C. Gonzaga, J. Daafouz, Min-switching stabilization for discrete-time switching systems with nonlinear modes, in: 4th IFAC Conference on Analysis and Design of Hybrid Systems ADHS'12, Eindhoven, the Netherlands, pp. 234-239. 
[26] M. Jungers, E. B. Castelan, S. Tarbouriech, J. Daafouz, Stabilization of discrete-time swithing systems including modal nonlinearities and saturation actuators, in: 3rd IFAC Conference on Analysis and Design of Hybrid Systems, Zaragoza, Spain, pp. 174-179.

[27] S. Tarbouriech, C. Prieur, J. M. Gomes da Silva Jr., Stability analysis and stabilization of systems presenting nested saturations, IEEE Transactions on Automatic Control 51 (2006) 1364-1371.

[28] J. C. Geromel, P. Colaneri, Stability and stabilization of discrete-time switched systems, International Journal of Control 79 (2006) 719-728.

[29] J. C. Geromel, P. Bolzern, P. Colaneri, Dynamic output feedback control of switched linear systems, IEEE Transactions on Automatic Control 53 (2008) 720-733. 